\section{Leishmaniose tegumentar americana: histórico, epidemiologia e perspectivas de controle}

\section{American cutaneous leishmaniasis: history, epidemiology and prospects for control}

\section{Sergio de Almeida Basano}

Centro de Medicina Tropical

Secretaria de Estado da Saúde de Rondônia

Postos do Correio de Monte Negro

78.965-970 Monte Negro/RO

sergio@icb5usp.med.br

Luís Marcelo Aranha Camargo

Departamento de Parasitologia

Instituto de Ciências Biomédicas

Universidade de São Paulo

Sub-Unidade de Monte Negro-Rondônia

\section{Resumo}

A Leishmaniose Tegumentar Americana (LTA) é uma doença causada por protozoários do gênero Leishmania, transmitida ao homem pela picada de mosquitos flebotomíneos (Ordem Diptera; Família Psychodidae; Sub-Família Phlebotominae). No Brasil existem atualmente 6 espécies de Leishmania responsáveis pela doença humana, e mais de 200 espécies de flebotomíneos implicados em sua transmissão. Trata-se de uma doença que acompanha o homem desde tempos remotos e que tem apresentado, nos últimos 20 anos, um aumento do número de casos e ampliação de sua ocorrência geográfica, sendo encontrada atualmente em todos os Estados brasileiros, sob diferentes perfis epidemiológicos. Estima-se que, entre 1985 e 2003, ocorreram 523.975 casos autóctones, a sua maior parte nas regiões Nordeste e Norte do Brasil.

Neste estudo, são discutidos aspectos relacionados ao tratamento e ao controle dessa doença, assim como também as dificuldades para a implementação dessas medidas. São apontadas alternativas que passam pela estruturação dos serviços de saúde, com respeito ao diagnóstico, no desenvolvimento de drogas de aplicação tópica ou por via oral, no desenvolvimento de vacinas, no controle diferenciado de vetores e no aprofundamento de estudos relacionados à biologia celular do parasita.

Palavras-chave: Leishmaniose tegumentar americana. Epidemiologia. Controle. Brasil. 
American Cutaneous Leishmaniasis (ACL) is an infectious disease transmitted by the bite of phlebotomines mosquitos (Order Diptera: Family Psychodidae: Sub-Family Phlebotominae) and caused by protozoa from the genus Leishmania (ROSS 1903). In Brazil, there are six different species of Leishmania and more than 200 different species of phlebotomines. It's a disease that has been afflicting human beings for many centuries, and in Brazil, in the past two decades, there has been an important increase in the number of cases and also in its geographical distribution. Presently, ACL cases are registered in all Brazilian states under three different epidemiological profiles (sylvatic, sylvaticmodified and periurban). Between 1985 and 2003, 523,975 cases were registered, mainly in the northeast and north of the country, although the north has the highest incidence rate.

In this study, aspects related to difficulties in treatment and transmission control, and new strategies to approach the disease are discussed. Alternatives such as the development of new drugs (topical and oral routes), the implementation of diagnostic methods in public health services, differentiated transmission control based on the 3 different epidemiological profiles and basic research on the molecular biology of the parasite are pointed out.

Key Words: American cutaneous leishmaniasis. Epidemiology. Control. Brazil.

\section{Histórico}

A leishmaniose tegumentar americana (LTA) é uma doença que acompanha o homem desde a antigüidade, existindo relatos e descrições encontrados na literatura desde o séc. I d.C. ${ }^{1,2}$.

Nas Américas, foram encontradas cerâmicas pré-colombianas, datadas de 400 a 900 anos d.C., feitas pelos índios do Peru, que apresentam mutilações de lábios e narizes, características da espúndia, hoje conhecida como leishmaniose cutânea-mucosa ${ }^{3}$. Posteriormente, através de estudos de paleomedicina, foram descobertas múmias com lesões de pele e mucosas características da leishmaniose ${ }^{4}$. A primeira referência de LTA no Brasil encontra-se no documento da Pastoral Religiosa Político-Geográfica de 1827, citado no livro de Tello intitulado "Antiguidad de la Syfilis en el Peru”, onde ele relata a viagem de Frei Dom Hipólito Sanches de Fayas y Quiros de Tabatinga (AM) até o Peru, percorrendo as regiões do vale amazônico ${ }^{5}$.

O primeiro a observar o parasita do gênero Leishmania foi Cunningham (1885), na Índia, em casos de leishmaniose visceral. No Brasil, Cerqueira, em 1855, observara a existência da moléstia da pele, identificando-a clinicamente como botão de Biskra. Em 1895, na Itália, Breda, descreveu a moléstia em italianos provenientes de São Paulo ${ }^{6}$.

Entretanto, no Brasil, a natureza leishmaniótica das lesões cutâneas e nasofaríngeas só foi confirmada, pela primeira vez, em 1909, por Lindenberg, que encontrou formas de Leishmania, idênticas à Leishmania tropica (Wright, 1903) da leishmaniose do Velho Mundo, em lesões cutâneas de indivíduos que trabalhavam nas matas do interior do Estado de São Paulo ${ }^{6}$.

Gaspar Vianna, por considerar o parasito diferente da $L$. tropica, o batizou de $L$. braziliensis, ficando assim denominado o agente etiológico da "úlcera de Bauru”, "ferida brava" ou "nariz de tapir"?

No Brasil, até a década de setenta, todos os casos de LTA eram atribuídos a $L$. braziliensis. Com o aprimoramento das técnicas de análise e a intensificação dos estu- 
dos ecológicos e epidemiológicos, outras espécies foram descritas, sendo registradas até o momento seis espécies causadoras da LTA $^{1,7,8-10}$.

\section{As leishmânias, seus vetores e reservatórios}

A LTA é uma doença não contagiosa, de evolução crônica, que acomete as estruturas da pele e cartilaginosas da nasofaringe, de forma localizada ou difusa, causada por várias espécies de protozoários digenéticos da ordem Kinetoplastida HONIGBERG, 1963, emend. VICKERMAN, 1976, família Trypanosomatide DOFLEIN, 1901 emend. GROBBEN, 1905 e do gênero Leishmania ROSS, 1903. Causam primariamente infecções de caráter zoonótico, acometendo o homem e seus animais domesticados de maneira secundária. A leishmaniose visceral, causada pela $L$. (V.) chagasi, que acomete o sistema linfomonocitário de forma sistêmica, muitas vezes com evolução fatal, não será abordada neste artigo.

As Leishmânias apresentam-se sob a forma amastigota em seus hospedeiros vertebrados. São estruturas arredondadas ou ovaladas sem flagelos, que parasitam o hospedeiro vertebrado em seu sistema linfomonocitário, alojando-se nos fagossomos dos monócitos, histiócitos e macrófagos onde vivem e se multiplicam por divisão assexuada até romperem a célula, disseminando-se pela via hematogênica e linfática ${ }^{11}$, iniciando uma reação inflamatória e proporcionando a atração de outros macrófagos gerando um ciclo vicioso ${ }^{12}$.

Os vetores, por sua vez, são mosquitos flebotomíneos (Ordem Díptera; Família Psychodidae; Sub-Família Phlebotominae), que sugam junto com o sangue as formas amastigotas de um animal infectado, que se alojam em partes de seu intestino levandoas a se transformar em promastigotas. Esta forma é alongada e apresenta um longo flagelo livre. No sistema digestivo de seus vetores, multiplicam-se por aparente divisão simples e assexuada e migram para a proboscíde do inseto após aproximadamente
4 a 5 dias. A esta altura, bloqueiam o proventrículo, de onde podem ser inoculadas na pele do hospedeiro vertebrado, junto com a saliva ${ }^{8}$.

No Brasil existem seis espécies destes parasitas causadores da Leishmaniose cutânea e estão divididas em 2 subgêneros: Leishmania e Viannia.

\section{Espécies de Leishmania envolvidas na etiologia da LTA no Brasil}

L. (Leishmania) amazonensis: Apresenta-se distribuída no Brasil, principalmente na bacia amazônica, em áreas de florestas primárias e secundárias tipo várzea e igapó (Amazonas, Pará, Rondônia e sudoeste do Maranhão), e também na Bahia, em Minas Gerais e em Goiás. Está presente em outros países como Colômbia, Paraguai, Bolívia e Guiana Francesa. O principal hospedeiro silvestre é o roedor Proechimys sp. (rato-soiá), além de outros roedores: Oryzomys sp., Neacomys sp., Nectomys sp e Dasyprocta sp.; os marsupiais Metachirus sp., Philander $s p$. , Didelphis sp. e Marmosa sp.; e a raposa Cerdocyon thous. Tem como principal vetor o Lutzomyia flaviscutellata, um flebotomíneo de hábito noturno e pouco antropofílico, e $\mathrm{Lu}$. olmeca nociva como vetor secundário no Amazonas e em Rondônia. É responsável no homem pela leishmaniose cutânea, cutâneo-mucosa, e por uma forma sem tratamento conhecida por leishmaniose cutânea difusa anérgica., ${ }^{1,-10}$

L. (Viannia) braziliensis: apresenta distribuição em todo o território nacional, além de vários países da América Central e do Sul (de Belize até a Argentina), sendo provável que nem todas correspondam exatamente ao mesmo parasita, mas que exista um complexo de subespécies. Existem poucas informações sobre seus hospedeiros silvestres, tendo sido relatado parasitas semelhantes em roedores (Akodon sp., Proechimys sp., Rattus sp., Oryzomys sp. e Rhipidomys sp.) e no marsupial Didelphis $s p$. Apresenta como vetor, na Serra dos Carajás, o Psychodopygus wellcomei (antropofílico, com picadas diuturnas e de 
maior atividade nas estações de chuva), além de outras espécies que vêm se adaptando em áreas peridomésticas, e ainda as de florestas primárias (Lu. whitmani, Lu. migonei, Lu. pessoai, $\mathrm{Lu}$. intermedia, $\mathrm{Lu}$. carrerai). $\mathrm{Na}$ transmissão peridoméstica, apresentam-se como hospedeiros: cavalos, cães, jumentos e até gatos. Causa no homem a leishmaniose cutânea e a cutâneo-mucosa ${ }^{1,7-10}$

L. (Viannia) guyanensis: distribuída ao norte do rio Amazonas no Brasil (Amazonas, Pará, Amapá e Roraima), e também em outros países como as Guianas, Peru, Equador e Venezuela. Tem como hospedeiros conhecidos a preguiça (Choloepus didactylus), o tamanduá (Tamandua tetradactyla), o marsupial Didelphis sp. e o roedor Proechimys sp., sendo transmitida pelos vetores $\mathrm{Lu}$. umbratilis (primariamente), Lu. anduzeie Lu. whitmani (secundariamente) ${ }^{10}$.

Estes vetores têm a característica de repousar em troncos das árvores em terra firme após o repasto, e quando perturbados de dia (principalmente) ou à noite, atacam em grande número, causando inúmeras lesões chamadas pian-bois (causadas também pela disseminação linfática). Causam no homem a Leishmaniose cutânea, com múltiplas lesões, e raramente lesões nas mucosas ${ }^{1,7-10}$.

L. (Viannia) lainsoni: distribui-se pela região Amazônica, sendo descrita até então no Pará, e em recente estudo em Rondônia (Basano, informação pessoal). Tem como hospedeiro a paca (Agouti paca), e como único vetor conhecido e de baixa antropofilia a Lu. ubiquitalis, causando com pouca freqüência a Leishmaniose cutânea ${ }^{1,7-10}$.

L. (Viannia) naiffi: distribui-se pelo Brasil nos Estados do Amazonas e Pará, e na Guiana Francesa. Tem como hospedeiro o tatu (Dasypus novemcinctus), sendo transmitido provavelmente por 3 espécies de flebotomíneos-Psychodopigus paraensis, Ps. ayrozai, Ps. Squamiventris - que apresentam alta antropofilia e cujos hábitos zoofílicos são pouco conhecidos. Causa no homem principalmente a Leishmaniose cutânea ${ }^{1,7-10}$.

L. (Viannia) shawi: encontrada no Estado do Pará, tem como hospedeiro os ma- cacos (Cebus apella, Chiropotes satanas), a preguiça "real", o "bentinha" (Choloepus didactylus e Bradypus tridactylus) e o quati (Nasua nasua), sendo o vetor uma espécie do complexo $L u$. whitmani, que tem como habitat natural os troncos de florestas primárias, atacando avidamente quando mo$\operatorname{lestados}^{1,7-10}$.

\section{Vetores: os flebotomíneos}

Os flebotomíneos (Ordem Díptera; Família Psychodidae; Sub-Família Phlebotominae) constituem um grupo deinsetos hematófagos, responsáveis pela transmissão das Leishmanioses. Geralmente não ultrapassam $0,5 \mathrm{~cm}$ de comprimento, tendo pernas longas e delgadas, e o corpo densamente piloso. Têm como característica o vôo saltitante e a manutenção das asas eretas, mesmo em repouso, ao contrário dos outros dípteros. Geralmente é de cor parda ("mosquito palha"), sendo que apenas as fêmeas estão adaptadas com o respectivo aparelho bucal para picar a pele de vertebrados e sugar o sangue. Apresentam vários nomes populares, variando segundo os países, Estados e regiões, a saber: mosquito palha, asa dura, asa branca, tatuquira, birigui, cangalha, cangalhinha, ligeirinho, péla-égua, arrupiado ${ }^{13}$.

O gênero Lutzomyia é o responsável pela transmissão das leishmanioses nas Américas, existindo 350 espécies catalogadas, distribuídas desde o sul do Canadá até o norte da Argentina. Destas, pelo menos 200 ocorrem na bacia amazônica ${ }^{13,14}$.

Muito pouco se sabe de seus criadouros, encontrando-se as formas imaturas em detritos de fendas de rocha, cavernas, raízes do solo e de folhas mortas e úmidas, e também nas forquilhas das árvores em tocas de animais - ou seja, em solo úmido, mas não molhado, e em detritos ricos em matéria orgânica em decomposição ${ }^{13}$.

\section{Distribuição geográfica e epidemiológica}

Estima-se que a Leishmaniose (tegumentar e visceral) hoje apresenta uma 
prevalência de 12 milhões de casos no mundo, sendo que 350 milhões de pessoas estão ameaçadas de contrair a doença em 88 países, 72 dos quais estão localizados em países em desenvolvimento. A saber, $90 \%$ de todos os casos de Leishmaniose visceral ocorrem no Brasil, Bangladesh, Índia e Sudão. Noventa por cento dos casos de leishmaniose cutâneo-mucosa ocorrem no Brasil, Bolívia e Peru, e 90\% dos casos de leishmaniose cutânea ocorrem no Brasil, Afeganistão, Irã, Peru, Arábia Saudita e Síria ${ }^{15}$.

Nas Américas, a LTA é considerada uma zoonose primária de mamíferos silvestres (roedores, marsupiais, edentados e primatas). Dessa forma, o homem adquire a infecção ao entrar em contato com as áreas florestais onde existem as enzootias pelas diferentes espécies de Leishmania. No Brasil, a LTA tem assegurada a sua distribuição em todo o território nacional, onde, atualmente todos os Estados federativos têm registros da enfermidade ${ }^{10}$, que pode se apresentar, basicamente, com três perfis ${ }^{16}$.

- Leishmaniose tegumentar puramente silvestre: ocorre através de surtos epidêmicos associados à derrubada das matas (construção de estradas, instalação de povoados em regiões pioneiras) e à exploração desordenada das florestas (extração de madeira, agricultura, mineração), ocorrendo na Amazônia de um modo geral.

- Leishmaniose tegumentar silvestre modificada: ocorre através de surtos epidêmicos sazonais, em áreas com pequenos focos residuais de mata primária. A infecção tem lugar na interface da área peridomiciliar e nas áreas de mata, onde o homem costuma desenvolver atividades ligadas à agricultura, estando ligada às flutuações da densidade populacional dos flebotomíneos e ocorrendo, por exemplo, no vale do rio São Francisco, Caratinga-MG e Viana-ES.

- Leishmaniose tegumentar periurbana: ocorre de forma endemo-epidêmica, endo ou peridomiciliar, em áreas de colonização antiga onde há suspeita da participação de animais domesticados como reservatórios (cães e eqüinos), ocorrendo em áreas da cidade do Rio de Janeiro.

\section{Distribuição da doença}

A incidência da LTA no Brasil não apresenta dados fidedignos e muito provavelmente está aquém do real, embora tenham sido feitas várias estimativas, dentre elas no período de 1980 a 1990, onde se chegou a um total de 154.103 casos $^{17}$. Outra estimativa, abrangendo os anos de 1985 a 1999, aponta 388.155 casos autóctones de LTA ${ }^{10}$. Comparando-se os valores absolutos e o coeficiente de detecção, houve um aumento de 13.654 casos/ano para 30.550 casos/ano, e de 10,45 casos/ 100.000 habitantes para 18,63 casos/ 100.000 habitantes nestes dois períodos, respectivamente ${ }^{10}$. Vários estudos brasileiros apontam para a ocorrência de LTA e seus vetores em todo o território brasileiro ${ }^{18-30}$, porém apresentando-se com diferentes coeficientes de incidência (Tabela 1). Deve-se salientar que a ocorrência do perfil periurbano de transmissão, está relacionada com a falta de saneamento básico, a situação econômica precária, a migração da população para as periferias das cidades, aos materiais de construção inadequados e ao convívio com animais ermos ou mesmo domesticados que servem de novos reservatórios da doença, aliados ao aumento da população de ratos que se concentram nos "depósitos" de lixo destas áreas.

Tabela 1 - Incidência de casos de LTA (x 100.000 habitantes), por macrorregião.

Table 1 - Incidence of ACL cases (x 100,000 inhabitants) by macro-region

\begin{tabular}{lccc}
\hline Região & 1997 & 1998 & 1999 \\
\hline Norte & 97,9 & 30,0 & 92,3 \\
Nordeste & 26,2 & 18,4 & 19,0 \\
Sudeste & 3,4 & 4,7 & 4,8 \\
Sul & 1,8 & 1,9 & 1,9 \\
Centro-Oeste & 44,2 & 27,1 & 50,4 \\
\hline
\end{tabular}

Fonte: Ministério da Saúde do Brasil, 2000 
Tabela 2 - Valores absolutos de LTA, por macrorregião

Table 2 - Number of cases of ACL, by macro-region

\begin{tabular}{lccccccc}
\hline Região & 1997 & 1998 & 1999 & 2000 & 2001 & 2002 & 2003 \\
\hline Norte & 11058 & 6078 & 11201 & 11140 & 12369 & 12489 & 14200 \\
Nordeste & 11868 & 8455 & 9112 & 13078 & 15057 & 11548 & 8005 \\
Sudeste & 2294 & 2945 & 3983 & 2938 & 2041 & 3514 & 3472 \\
Sul & 430 & 455 & 460 & 853 & 635 & 1053 & 951 \\
Centro-Oeste & 4640 & 2981 & 6384 & 4605 & 4962 & 5440 & 4635 \\
\hline
\end{tabular}

Fonte: Ministério da Saúde do Brasil, 2004

\section{Diagnóstico laboratorial}

O diagnóstico laboratorial baseia-se principalmente na pesquisa de parasitas em esfregaço das lesões após a coloração de Giemsa. Utiliza-se para a coleta do material a escarificação do bordo da lesão, biópsia do bordo com impressão por aposição em lâmina de vidro e/ou punção aspirativa da lesão. Pode-se realizar o diagnóstico de forma indireta, através da mensuração da resposta imune celular com a injeção intradérmica de antígenos do parasita (intradermorreação de Montenegro), que deve ser medida em 48 a 72 horas, sendo consideradas positivas as reações com área de enduração maior que $5 \mathrm{~mm}$. Em laboratórios de maior complexidade, podem ser realizados outros exames, tais como histopatológico (coloração pela hematoxilina-eosina e uso de anticorpos marcados com imunoperoxidase para detecção do parasita), cultivo in vitro (meio $\mathrm{NNN}$ bifásico), in vivo (hamster dourado), sorologia (imunofluorescência, ELISA e aglutinação direta) e PCR (reação da polimerase em cadeia) ${ }^{2,31}$, com resultados em geral satisfatórios ${ }^{32,33}$, porém de pouco valor na prática diagnóstica em áreas endêmicas. Na prática, a conduta mais utilizada é a pesquisa direta de parasitas e a intradermorreação $0^{2,10,34}$, aliadas ao aspecto clínico da lesão e ao antecedente epidemiológico.

\section{Diagnóstico diferencial}

Nas lesões cutâneas, devem ser excluídas as úlceras traumáticas, as de estase, as tropicais, as por diabetes, as por anemia falciforme, as piodermites, paracoccidiodomicose, esporotricose, cromoblastomicose, neoplasias cutâneas, sífilis e tuberculose, devendo a hanseníase virchowiana ser incluída quando se tratar de leishmaniose cutânea difusa.

Nas lesões mucosas, devem ser excluídas a paracoccidiodomicose, hanseníase virchowiana, rinoscleroma, sarcoidose, bouba, sífilis terciária, granuloma médio facial e neoplasias ${ }^{9,31,34,35}$.

\section{Tratamento}

A droga de primeira escolha é o antimonial pentavalente, que foi padronizado pela Organização Mundial da Saúde na dose entre 10 a $20 \mathrm{mg} / \mathrm{Sb}^{+5} / \mathrm{Kg} /$ dia $\left(\mathrm{Sb}^{+5}\right.$ significando antimônio pentavalente), por 20 a 30 dias. Há drogas alternativas como stibugluconato de pentamidina e anfotericina $\mathrm{B}$, utilizadas nas formas rebeldes ao tratamento convencional. Vale enfatizar que, mesmo com o tratamento adequado, a ocorrência de recidivas e/ou comprometimento mucoso é freqüente, sendo de $2 \%$ nos casos tratados e ao redor de $10 \%$ nos casos não tratados ${ }^{10,34,36}$.

Algumas drogas administradas de forma tópica ou por via oral têm apresentado resultados encorajadores, muito embora sejam necessários testes clínicos adicionais para se conhecer sua real eficácia. Vale ressaltar o papel do miltefosine, utilizado por via oral em doses de 133 e 150mg ao dia por 4 semanas, que obteve índices de cura da ordem de 100 e $89 \%$, respectivamente ${ }^{37}$. Estudos preliminares com o fluconazol no tratamento de leishmaniose causada por $L$. major (que não ocorre no Brasil) indicam 
uma eficácia de $79 \%$ quando comparado ao placebo $(34 \%)^{38}$.

O uso de produtos tópicos como paramomycin e imiquimod, associados ou não à medicação parenteral, têm igualmente apresentado resultados preliminares satisfatórios, com índices de cura variando entre $74 \% \mathrm{e}$ $85 \%$ para a primeira droga, e $90 \%$ para a segunda $^{39,40}$.

\section{Perspectivas de controle}

O controle da LTA deve ser abordado, de maneira abrangente, sob cinco aspectos: vigilância epidemiológica, medidas de atuação na cadeia de transmissão, medidas educativas, medidas administrativas e vacina. A vigilância epidemiológica abrange desde a detecção do caso, a sua confirmação, o registro de sua terapêutica, o registro das variáveis básicas, fluxo de atendimento e informação, até finalizar com as análises de dados distribuídos em indicadores epidemiológicos (casos autóctones em valores absolutos e os coeficientes gerais e proporcionais) e indicadores operacionais (proporção de métodos diagnósticos auxiliares, cura, abandono e tratamento regular), visualizando e caracterizando a distribuição da doença e de seu perfil clínico e epidemiológico.

As medidas de atuação, em virtude de suas peculiaridades, devem ser flexíveis e distintas, baseadas nas características epidemiológicas em particular, aliadas a um sistema de saúde básico capacitado para diagnóstico precoce e tratamento adequado. Nas áreas de maior incidência, as equipes do Programa Saúde da Família podem ter importante papel na busca ativa de casos e na adoção de atividades educacionais junto à comunidade. Nas áreas de perfil periurbano ou de colonização antiga deve-se tentar a redução do contato vetorial através de inseticidas de uso residual (onde houver a notificação de casos em menores de 10 anos), do uso de medidas de proteção individual como mosquiteiros, telas finas nas janelas e portas, repelentes e roupas que protejam as áreas expostas, e de distanciamento mínimo de 200 a 300 metros das moradias em relação à mata. Outra estratégia de controle seria a abordagem dos focos de transmissão peridomiciliar, implementando as condições de saneamento para evitar o acúmulo de lixo e de detritos que possam atrair roedores e pequenos mamíferos, somadas as melhorias das condições habitacionais ${ }^{9,10,34}$. Aliadas a estas medidas deveriam ser valorizadas as atividades de capacitação continuada dos profissionais de saúde em todos os seus níveis. Por fim, como medida definitiva, o uso de uma vacina eficiente e operacional.

\section{Principais dificuldades}

O despreparo das unidades de saúde para o diagnóstico de LTAé, sem dúvida, um grande obstáculo para uma abordagem precoce do doente. Normalmente, a maior parte dos serviços de saúde não está capacitada a realizar a pesquisa de parasitas em esfregaço da lesão e/ou não possuem o antígeno de Montenegro para se aplicar a intradermoreação. Dados preliminares de Rondônia apontam para um tempo médio de evolução da lesão leishmaniótica da ordem de 15 meses, caracterizando, portanto, um longo período entre o início da lesão e o diagnóstico da doença, ocasionado, em parte, pela falta de capacidade diagnóstica e pela falta de capacitação técnica dos profissionais de saúde (Basano, informação pessoal). Outros métodos diagnósticos como técnicas sorológicas, histopatológico e PCR, ou ainda se encontram em fase experimental e/ou não são aplicáveis no âmbito do sistema básico de saúde. Seriam desejáveis esforços no sentido de disponibilizar minimamente os métodos diagnósticos tradicionais (pesquisa de parasita pela microscopia e intradermorreação de Montenegro) a todas as unidades de saúde em áreas de transmissão, incrementar as atividades de capacitação para diagnóstico de LTA, e criar um sistema de referência para a realização de exames mais complexos para casos clínicos específicos, como nas lesões crônicas, recidivas ou nas formas mucosas, geralmente de diagnóstico mais difícil.

Com relação à terapêutica, muito em- 
bora a droga esteja geralmente disponível nas unidades de saúde, seu potencial hepato, cardio e nefrotóxico do antimonial, aliado ao seu uso parenteral exclusivo, representa um sério obstáculo ao tratamento adequado dos casos. Deve-se ter em conta que a maioria dos casos ocorre em áreas de difícil acesso, em meio à floresta, o que dificulta tanto a aplicação parenteral da droga como o monitoramento de seus efeitos colaterais. A terapêutica da LTA, alicerçada nas drogas disponíveis atualmente, representa, per si, um obstáculo à condução clínica adequada dos casos de LTA. Esforços devem ser efetivados no sentido de ampliar os testes clínicos com as novas drogas promissoras por via oral (fluconazol e miltefosine), além das drogas de uso tópico (paromomycin e imiquimod), e pesquisar drogas alternativas que facilitem o tratamento.

Os diferentes perfis epidemiológicos com os quais a LTA se apresenta sugerem medidas de controle de transmissão diferenciadas.

$\mathrm{Na}$ forma de transmissão silvestre pura ou modificada, as ações de controle são mais difíceis ou não aplicáveis frente ao caráter zoonótico da parasitose. Neste caso, poderse-ia apostar em uma vacina eficiente e operacional, proporcionando ou não imu- nidade esterilizante. Apesar da abundância de estudos neste sentido, e de alguns resultados otimistas ${ }^{41-49}$, não se espera, a curto prazo, a disponibilização deste importante instrumento, continuando a ser problemático o controle da endemia com este perfil de transmissão. Neste caso, a oferta de um sistema de saúde eficiente no diagnóstico e condução clínica dos casos, seria uma alternativa para abrandar os agravos causados pela endemia.

Com relação ao perfil de transmissão periurbano, além das medidas antivetoriais e de um eficiente sistema de vigilância epidemiológica, a redução da transmissão está intimamente relacionada com a melhoria das condições de vida da população, problemática que foge ao escopo técnico da área de saúde, representando atualmente mais um obstáculo na abordagem do controle de endemias.

\section{Agradecimentos}

Aos doutores Ricardo Augusto Fagundes Lopes e Sati Jaber Mahmud, pelo auxílio na pesquisa bibliográfica, à bióloga Denise da Silva Rezende e à Profa. Filomena Brondani pela revisão do texto.

\section{Referências}

1. Lainson R. Leishmânia e leishmaniose, com particular referência à região Amazônica do Brasil. Revista Paraense de Medicina 1997; 11(1): 29-40.

2. Camargo LMA, Barcinski MA Leishmanioses, feridas bravas e kalazar. Ciência e Cultura 2003, 1:34-7.

3. Lainson R, Shaw JJ. New world Leishmaniasis - The Neotropical Leishmania species. In: Topley \& Wilson. Microbiology and Microbial Infections (9 a ed). London: Ed. Feg Cox; 1988.

4. Santos RV, Coimbra JRCEA. Saúde e Povos Indígenas Rio de Janeiro: 1994, Ed. FIOCRUZ; 1994.

5. Paraguassu-Chaves CA. Geografia Médica ou da Saúde (Espaço e doença na Amazônia Ocidental). Rondônia: Edufro; 2001.

6. Pessôa SM. Parasitologia Médica. Rio de Janeiro: Guanabara Koogan; 1982.
7. Silveira FT, Lainson R, Brito AC, Oliveira MRF, Paes MG, Souza AAA, Silva BM. Leishmaniose Tegumentar Americana. In: Leão RNQ. Doenças Infecciosas e Parasitárias: Enfoque Amazônico. Belém: Editora CEJUP; 1997.

8. Marzochi MCA. Leishmanioses no Brasil (As Leishmanioses Tegumentares). JBM 1992; 63 (5/6): 81105 .

9. Ministério da Saúde do Brasil. Manual de Controle da Leishmaniose Tegumentar Americana. Brasília; 1994.

10. Ministério da Saúde do Brasil. Manual de Controle da Leishmaniose Tegumentar Americana. Brasília; 2000.

11. Lainson R, Shaw JJ. A brief history of genus Leishmania (Protozoa: Kinetoplastida) in the Americas with particular reference to Amazonian Journal of the Brazilian Association for the Advancement of Science 1992; 44: 94-106. 
12. Raso P, Genaro O. Leishmaniose Tegumentar Americana. In: Brasileiro Filho, G et al. Bogliolo Patologia. Rio de Janeiro: Guanabara Koogan, 1994.

13. Rebêlo JMM (b). Flebótomos vetores das leishmanioses (Manual para técnicos e profissionais de Saúde) São Luis: Universidade Federal do Maranhão/ Ministério da Saúde; 1999.

14. Gil LHS, Basano SA, Souza AA, Silva MGS, Barata I, Ishikawa EA, Camargo LMA et al. Recent observations on the sand fly (Diptera:Psychodidae) fauna of the sate of Rondônia, Western Amazônia, Brazil: the importance of Psychodopygus davisi as a vector of zoonotic cutaneous leishmaniasis. Mem Inst Oswaldo Cruz 2003; 98: 751-5.

15. (http: /www.who.int/emc/disease/leish/index.html) [falta data do acesso e título]

16. Silveira, FT. Patogenia da leishmaniose tegumentar americana: caracterização clínica, histopatológica e imunológica da leishmaniose disseminada, com ênfase na Leishmania (L.) amazonensis. Tese de doutoramento. São Paulo: Faculdade de Medicina da USP; 2001.

17. World Health Organization. Control of leishmaniases: Report of WHO expert committee. Technical report series 793. Geneva; 1990.

18. Grisard EC, Steindel M, Shaw JJ, Ishikawa EA, Carvalho-Pinto CJ, Eger-Mangrich I et al. Characterization of Leishmania sp. strains isolated from autochthonous cases of human cutaneous leishmaniases in Santa Catarina State, southern Brazil. Acta Tropica 2000; 74: 89-93.

19. Silveira TG, Teodoro U, Lonardoni MV, Guilherme AL, Toledo MJ, Ramos M et al.. Epidemiologics aspects of cutaneous leishmaniasis in an endemic area of the state of Paraná, Brazil. Cad Saude Publica 1996; 12: 141-7.

20. Tedoro U, Kühl JB.Interação flebotomíneos, animais domésticos e dominância de Lutzomyia (Nyssomyia) intermedia (Lutz \& Neiva, 1912) em área com alto grau de antropia, no Sul do Brasil. Rev Saúde Pública 1997; 31: 512-6.

21. Luz E, Membrive N, Castro EA, Dereure J, Pratlong F, Dedet JÁ et al. Lutzomyia whitmani (Diptera: Psychodidae) as vector of Leishmania (V.) braziliensis in Paraná State, southern Brazil. Am Trop Med Parasito 2001; 94: 623-31.

22. Giorgio S, Deberaldini ER, Pacheco RS, Pires MQ, ZanottiMagalhães EM, Cordeiro N et al. Cases of american cutaneous leishmaniasis caused by Leishmania (Viannia) braziliensis in the towns of Cosmopolis and Indaiatuba - region of Campinas, in Sao Paulo, Brazil. Rev Soc Bras Med Trop 1996; 29: 419-24.

23. Domingos MD, Carreri-Bruno GC, Ciaravolo RD, Galati EA , Wanderley DM , Corrêa FM. American tegumentary leishmaniasis: Phlebotominae in an area of disease transmission, city of Pedro de Toledo, southern region of the state of Sao Paulo, Brazil. Rev Soc Bras Med Trop 1998; 31: 425-32.
24. Aguiar GM, Medeiros WM, De Marco TS, Santos SC, Gambardella S. Ecology of sandflies in Serra do Mar, Itaguaí, state of Rio de Janeiro, Brazil. I - Sandfly fauna and prevalence of the species in collections sites and method of capture. Cad Saude Publica 1996; 12: 195206.

25. Galati EA, Nunes VL, Dorval ME, Oshiro ET, Cristaldo G, Espíndola MA et al. Study of the phlebotomines (Diptera, Psychoddidae), in area of cutaneus leishmaniasis in the State of Mato Grosso do Sul, Brazil. Rev Saude Publica 1996; 30: 115-28.

26. Azevedo AC, Vilela ML, Souza NA, Andrade-Coelho CA, Barbosa AF, Firmo AL et al. The sand fly fauna (Diptera: Psychodidae: Phlebotominae) of a focus of cutaneuos leishmaniais in Ilheus, state of Bahia, Brazil. Mem Inst Oswaldo Cruz 1996; 91: 75-9.

27. Follador I, Araújo C, Cardoso MA, Neto JT, Barral A, Miranda JC et al. Surto de leishmaniose tegumentar americana em Canoa, Santo Amaro, Bahia, Brasil. Rev Soc Bras Med Trop 1999; 32: 497-503.

28. Sousa AD, Parise ME, Pompeu MM, Coehlo Filho JM, Vasconcelos IA, Lima JW et al. Bubonic leishmaniasis: a common manifestation of Leishmania (Viannia) braziliensis infection in Ceara, Brazil. Am J Trop Med Hyg 1995; 53: 380-5.

29. Brandão-Filho SP, Campbell-Lendrum D, Brito ME, Shaw JJ, Davies CR. Epidemiological surveys confirm an increasing burden of cutaneous leishmaniasis in northeast Brazil. Trans R Soc Trop Med Hyg 1999; 93: 488-94.

30. Balbino VQ, Marcondes CB, Alexander B, Luna LK, Lucena MM , Mendes AC et al. First report of Lutzomyia (Nyssomyia) umbratilis Ward \& Frahia, 1977 outside the Amazonian Region, in Recife, state of Pernambuco, Brazil (Diptera: Psychodidae: Phlebotominae). Mem Inst Oswaldo Cruz 2001; 96: 315-7.

31. Singh S, Sivakumar R. Recent advances in the diagnosis of leishmaniasis. J Postgrad Med 2003; 49: 55-60.

32. Faber WR; Oskam L, van Gool T, Kroon NC, Knegt-Junk $\mathrm{KJ}$, Hofward $\mathrm{H}$ et al. Value diagnostic techniques for cutaneous leishmaniasis. J Am Acad Dermatol 2003; 49: $70-4$.

33. Vega-Lopez F. Diagnosis of cutaneous leishmaniasis. Curr Opin Infect Dis 2003; 16: 97-101.

34. Ministério da Saúde do Brasil. Fundação Nacional de Saúde. In: Guia de Vigilância Epidemiológica 2002; 2: 501-24.

35. Montenegro J. Cutaneous reactions in leishmaniasis. Archives of Dermatology and Syphilology 1926; 13: 187.

36. Marsden, PD. Mucosal leishmaniasis ("Espundia”, Escomel, 1911). Trans R Soc Trop Med Hyg 1986; 80: 859-76. 
37. Jha TK, Sundar S, Thakur CP, Bachmann P, Karbwang J, Fischer C et al. [falta o nome do artigo]. N Engl J Med 1999; 341:1795-1800.

38. Alrajhi AA, Ibrahim EA, de Vol EB, Khairat M, Faris RM, Maguire JH. Fluconazol for the treatment of cutaneous leishmaniasis caused by Leishmania major. $N$ Engl $J$ Med 2002, 346: 891-5.

39. Davies CR, Kaye P, Croft SL, Sundar S. Leishmaniasis: a new approach to disease control. BMJ 2003; 326: 37782.

40. Arevalo I, Ward B, Miller R, Meng TC, Najar E, Alvarez E et al. Successful treatment of drug-resistant cutaneous leishmaniasis in humans by use of imiquimod, an immunomodulator. Clin Infect Dis 2001; 33: 1847-51.

41. Masina S, Gicheru MM, Demotz SO, Fasel NJ Protection against cutaneous leishmaniasis in outbred vervet monkeys using a recombinant histone antigen. $J$ Infect Dis 2003; 188: 1250-7.

42. Mendez S, Tabbara K Belaid Y, Bertholet S, Verthelyi D, Klinman D et al. Coinjection with Cp-G-containing immunostimulatory oligodeoxynucleotides reduces the pathogenicity of a live vaccine against cutaneous leishmaniasis but maintains its potency and durability. Infect Immun 2003; 71: 5121-9.

43. Pinto EF, de Mello Cortezia M, Rossi-Bergmann B. Interferon-gamma-inducing oral vaccination with Leishmania amazonensis protects BALB/c and C57BL/ 6 mice against cutaneous leishmaniasis. Vaccine 2003; 21: 3534-41.
44. Reed SG, Campos-Neto A. Vaccines for parasitic and bacterial diseases. Curr Opin Immunol 2003; 15: 45660 .

45. Reed SG, Coler RN, Campos-Neto A. Development of a lesihmaniasis vaccine: the importance of MPL. Expert Rev Vaccines 2003; 2: 239-52.

46. Cardoso SR, da Silva JC, da Costa RT, Mayrink W, Melo $\mathrm{MN}$, Michalic MS et al. Identification and purification of immunogenic proteins from nonliving promastigote polyvalent Leishmania vaccine (Leishvacin). Rev Bras Med Trop 2003; 36: 193-9.

47. Daneshvar H, Coombs GH, Hagan P, Phillips RS. Lesihmania mexicana and Leishmania major: attenuation of wild-type parasites and vaccination with attenuated lines. $J$ Infect Dis 2003; 187: 1662-8.

48. Manuel J. Vacinatoin against Leishmania infections. Curr Drug Targets Immune Endocr Metabol Disord 2002; 2: 201-26.

49. Melby PC. Vaccination against cutaneous leishmaniasis: current status. Am J Clin Dermatol 2002; 3: $557-70$

recebido em: 31/10/03 versão reformulada apresentada em: 27/08/04 aprovado em: 20/09/04 